\title{
MULTIPLE RESOURCE MODELING OF TASK INTERFERENCE IN VEHICLE CONTROL, HAZARD AWARENESS AND IN-VEHICLE TASK PERFORMANCE
}

\author{
William J. Horrey and Christopher D. Wickens \\ Aviation Human Factors Division \\ University of Illinois \\ Urbana, IL USA \\ E-mail: horrey@s.psych.uiuc.edu
}

\begin{abstract}
Summary: We describe a computational model of multiple task performance used to predict task interference and subsequent decrements in performance, based on the resource demands of a particular task (i.e., the difficulty) as well as the competition between tasks over limited and overlapping resources. We describe the model components, the computational aspects, and further validate it with data from a simulated driving study.
\end{abstract}

Successful multiple resource models predict the amount of interference that will occur between two or more tasks that are performed concurrently. That is, they predict the degradation in performance in one or multiple tasks when they are performed at the same time compared to their relative performance when performed alone (single-task conditions). Designers would like to be able to predict such interference in advance of time-consuming driver-in-the-loop simulations in order to assess, for example, whether a particular in-vehicle technology (IVT) will excessively interfere with safe driving.

Theories of multiple resources (Navon \& Gopher, 1979; Wickens, 2002) posit the existence of separate resources, which are both limited in capacity and allocatable amongst different tasks. In keeping with their "multiple" nature, these resources are defined along four dimensions: the information processing stage (perception / cognition vs. response), the processing code (verbal vs. spatial), the input modality (visual vs. auditory), and the visual channel (within the visual modality; focal vs. ambient). Resource competition is derived from interference at each of these levels. In general, theories of multiple resources predict greater task interference decrements when multiple tasks compete for limited and overlapping resources and when task difficulty is increased, as opposed to conditions that are easy in nature or combine tasks that draw on nonoverlapping resource structures. Task prioritization strategies will determine the nature of the performance decrement across multiple tasks.

Here we describe and test a computational model of multiple task performance in driving, based on the difficulty (or the resources demanded) of each task and the extent to which two concurrent tasks compete for common resources.

\section{INTERFERENCE MODEL}

Our computational model of task interference (Wickens, 2002) involves a number of steps, including the development of a demand vector and conflict matrix, followed by the calculation of the total task interference: 
(1) Each task is coded in terms of its dependence on a given resource (following the dimensions noted above) on an ordinal scale, depending on task characteristics and overall difficulty. That is, we assume that a value of 0 indicates that a given task does not involve a particular resource, whereas a value of 1 implies that some resources are demanded. As the task becomes more complex or difficult, this demand value may increase to 2, 3 or beyond. As a simple example, the task of keeping one's vehicle in its respective lane may involve resources at the perceptual (e.g., localizing the lane markers), cognitive (e.g., determining the relative position of the vehicle within the lane), and response (e.g., turning the steering wheel) levels. The relatively easy task of driving on a straight, uncluttered freeway may involve a resource demand vector of 1-1-1 along each of these dimensions, respectively. In contrast, driving at night on the same road may yield a demand vector of 2-1-1 along the same dimensions. A demand vector of 2-1-0 may characterize an in-vehicle task, such as perceiving and understanding a navigation display. The distribution of zero and non-zero values in the demand vector becomes an important consideration when we discuss the conflict matrix, below.

(2) The degree of task difficulty is assessed using a demand scalar, which is simply the additive combination of values in the demand vector. In the previous examples, the respective driving tasks would have demand scalars of 3, 4, and 3. When two tasks are performed concurrently, we derive the total demand score by summing the demand scalars for each task. For example, concurrent performance of the IVT task and the easy driving task would yield a total demand score of $(3+3=) 6$. In our model, we transform these scores for a given set of task combinations such that scores range from 0 (easiest combination) to 1 (most challenging).

(3) The extent to which a set of tasks shares overlapping resources is characterized along the four dimensions of the model. That is, we determine whether there is competition over visualspatial resources, over visual-verbal resources, and so on. We then establish how much interference is present within a given resource, based on the summed conflict values for the specified task set. As shown in Table 1, a conflict matrix is a convenient means of illustrating the conflict values for different resource competitions. We assume that if two tasks cannot be time-shared by a given resource, they will receive the maximum conflict value of 1 (e.g., two tasks requiring simultaneous delivery of a vocal response). In contrast, two tasks that can time-share the resource perfectly will receive a conflict value of 0 ; we assume, however, a default conflict value of 0.2 (e.g., a fundamental cost of concurrence). In this way, the conflict value in a particular cell increments as more resources are shared between the task vectors, defined by its rows and columns - thus leading to greater interference for identical resources (i.e., along the negative diagonal; see Wickens, 2002) ${ }^{1}$.

Using the values in the conflict matrix, we determine the resource-conflict score by transposing the demand vector for each task along the rows and columns of the table (i.e., the bold-faced vectors in Table 1). Whenever a cell in the matrix is occupied by non-zero demands from both tasks, it will contribute to the resource-conflict score by an amount equal

\footnotetext{
${ }^{1}$ Although the complete version of the multiple resource model nests the focal-ambient distinction within the visual perceptual resources, for the present analysis we have made the following simplification: because ambient vision is essentially spatial vision, and focal vision encompasses the recognition of both objects and symbols (digits/letters), the current model defines a single dichotomy of ambient-spatial versus focal-object-symbol.
} 
to the cell value. For example, the IVT and driving tasks both demand focal-visual resources ( 1 and 2, respectively). As such, the cell conflict value of 0.8 is added to the resource-conflict score. In contrast, there is no competition for spatial response resources ( 1 and 0$)$; therefore there would be no additional conflict along this dimension. Again, in our model we transform the total resource-conflict scores for a set of task combinations.

Table 1. Adapted conflict matrix, based on dimensions of the multiple resource model, including perceptual, cognitive, and response (based on Wickens, 2002). $\mathrm{V}=\mathrm{Visual}, \mathrm{A}=$ Auditory, $\mathrm{C}=$ Cognitive, $\mathrm{R}=$ Response, $\mathrm{f}=$ Focal, $\mathrm{a}=$ Ambient, $\mathrm{s}=$ Spatial, $\mathrm{v}=$ Verbal. The boldfaced numbers represent demand vectors (Ex.) for the sample IVT (vertical) and easy driving tasks (horizontal; described previously).

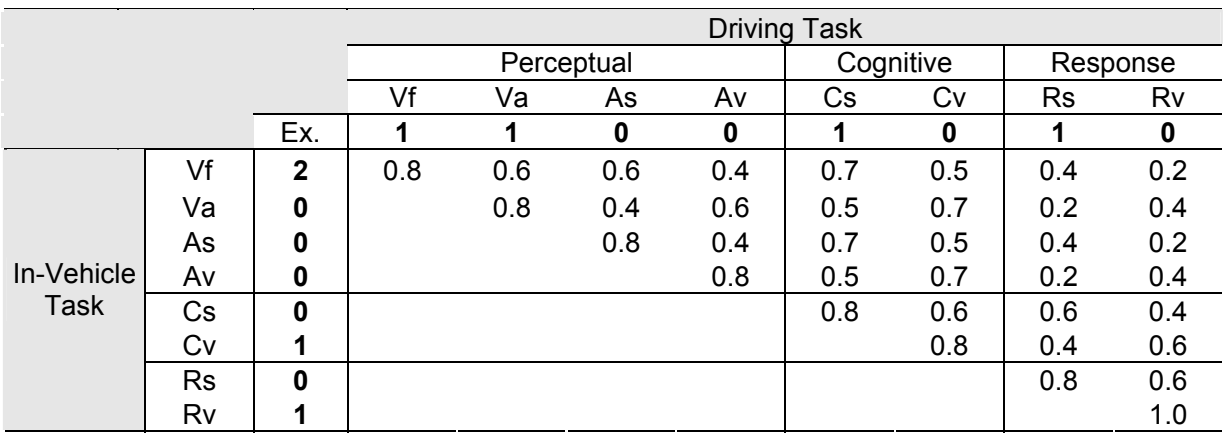

(4) The total interference score is represented by the sum of the total demand score and the resource-conflict score. As such, this total interference value is sensitive to structural interference from resource competition (and does not discriminate the source of the interference across the two tasks) as well as to the overall task difficulty. The predicted total interference can then be apportioned to either or both of the competing tasks, depending on the extent to which one or the other is treated as "primary" or "secondary."

\section{VALIDATION STUDY}

Using data from a simulated driving study, we evaluated the task demands and interference for nine different combinations of driving conditions (primary task difficulty) and in-vehicle technology (IVT) interfaces (secondary task). Results from this study are presented elsewhere (Horrey \& Wickens, 2002). Here we present a brief overview of the methodology and limit our discussion to the performance decrements in dual-task (relative to single-task) conditions.

Twenty-five drivers in a fixed-base wrap-around simulator drove urban and rural routes of varying complexity while engaging in a secondary phone number read-back task presented by different displays. Priority was given to safe vehicle control and avoidance of road hazards. The secondary task was presented in one of two modalities: either in a visual display located in a head-up display (HUD; $7^{\circ}$ below the horizon line) or on a head-down display (HDD; located $38^{\circ}$ offset from the forward horizon, near the mid-console), or auditorily through the car speakers. When the phone numbers were seen or heard, drivers were asked to read back the digits as quickly and accurately as possible. Digit strings were 4, 7, or 10 digits in length. Driver performance measures of lane keeping and speed control were recorded. IVT task performance was measured by response time to the digits, response duration, and the response accuracy. 
Additionally, we measured response times to discrete road hazard events that occurred periodically throughout the experimental trials.

In following the model, we established demand vectors for the three different types of driving complexity, as well as for the different IVT display configurations. These demand vectors are shown in Table 2. For example, city environments demanded more visual-focal resources than did straight rural roads; visual IVT displays required some degree of focal resources, whereas none were required in the auditory IVT condition.

Table 2. Demand vectors for the driving and IVT tasks in Horrey \& Wickens (2002).

\begin{tabular}{|c|c|c|c|c|c|c|c|c|c|}
\hline \multirow{3}{*}{ Task } & \multicolumn{8}{|c|}{ Demand Vector } & \multirow{3}{*}{$\begin{array}{c}\text { Demand } \\
\text { Scalar }\end{array}$} \\
\hline & \multicolumn{4}{|c|}{ Perceptual } & \multicolumn{2}{|c|}{ Cognition } & \multicolumn{2}{|c|}{ Response } & \\
\hline & $\mathrm{Vf}$ & $\mathrm{Va}$ & As & Av & $\mathrm{Cs}$ & $\mathrm{Cv}$ & Rs & $\mathrm{Rv}$ & \\
\hline (A) City Driving & 2 & 1 & 0 & 0 & 2 & 0 & 1 & 0 & 6 \\
\hline (B) Rural Straight Driving & 1 & 1 & 0 & 0 & 1 & 0 & 1 & 0 & 4 \\
\hline (C) Rural Curved Driving & 1 & 2 & 0 & 0 & 1 & 0 & 2 & 0 & 6 \\
\hline (D) IVT HUD Adjacent & 1 & 0 & 0 & 0 & 0 & 1 & 0 & 1 & 3 \\
\hline (E) IVT HDD Console & 2 & 0 & 0 & 0 & 0 & 1 & 0 & 1 & 4 \\
\hline (F) IVT Auditory & 0 & 0 & 0 & 2 & 0 & 2 & 0 & 2 & 6 \\
\hline
\end{tabular}

As described in the previous section, the demand scalars from Table 2 were summed across each different concurrent task combination (e.g., (a) city driving with (d) IVT HUD, (e) IVT HDD, and with (f) IVT auditory side tasks) and transformed, such that values were bounded on a ratio scale between 0 and 1 . The demand vectors for concurrent tasks were then used to determine the overlapping cells within the conflict matrix shown in Table 1. The transformed set of resourceconflict scores for the nine task combinations was then added to the respective demand scores to yield the model-predicted interference scores.

We then compared these predicted interference scores with actual scores for a subset of measures, including lane keeping (i.e., vehicle control), response times to the IVT task, and response times to the critical traffic hazards. We selected these measures as representative of the required (concurrent) tasks. To obtain the actual interference score, we compared performance of the relevant task in dual-task conditions (i.e., driving plus IVT) with performance in single-task conditions (e.g., driving alone). Thus, interference was expressed as a performance decrement.

Finally, we plotted the obtained interference values as a function of the predicted values for each of the different measures and followed with a model-fitting exercise. As shown in Figure 1, the regression analysis of obtained values on predicted interference revealed varying degrees of fit across the different performance measures. We were able to predict $85 \%$ of the variance in performance decrements in secondary task latency and $98 \%$ of the variance in response times to critical road hazards (a focal-visual task). (Only three data points were used in the latter case because of the rarity of these events; these were averaged over road type.) The resource model, however, did not predict variance in the task of lane keeping $\left(\mathrm{R}^{2}=0.02\right)$, suggesting that drivers were optimal in their resource allocation by protecting the continuous aspects of vehicle control from the differential resource competition offered by the different conditions. Rather, interference was manifested in degraded IVT side-task performance and in hazard detection. 


\section{DISCUSSION}

We have shown that this computational model can be used to predict performance decrements in different combinations of dual-task conditions. Even in those cases where the model does not predict significant levels of variance (i.e., for lane keeping), we can extrapolate important information-specifically, that drivers were prioritizing this task, such that all variance in the performance decrements would be borne by the IVT task (as was shown). However, this protection was not generalizable to all aspects of the driving task, as there were performance decrements in the important task of hazard detection and response.
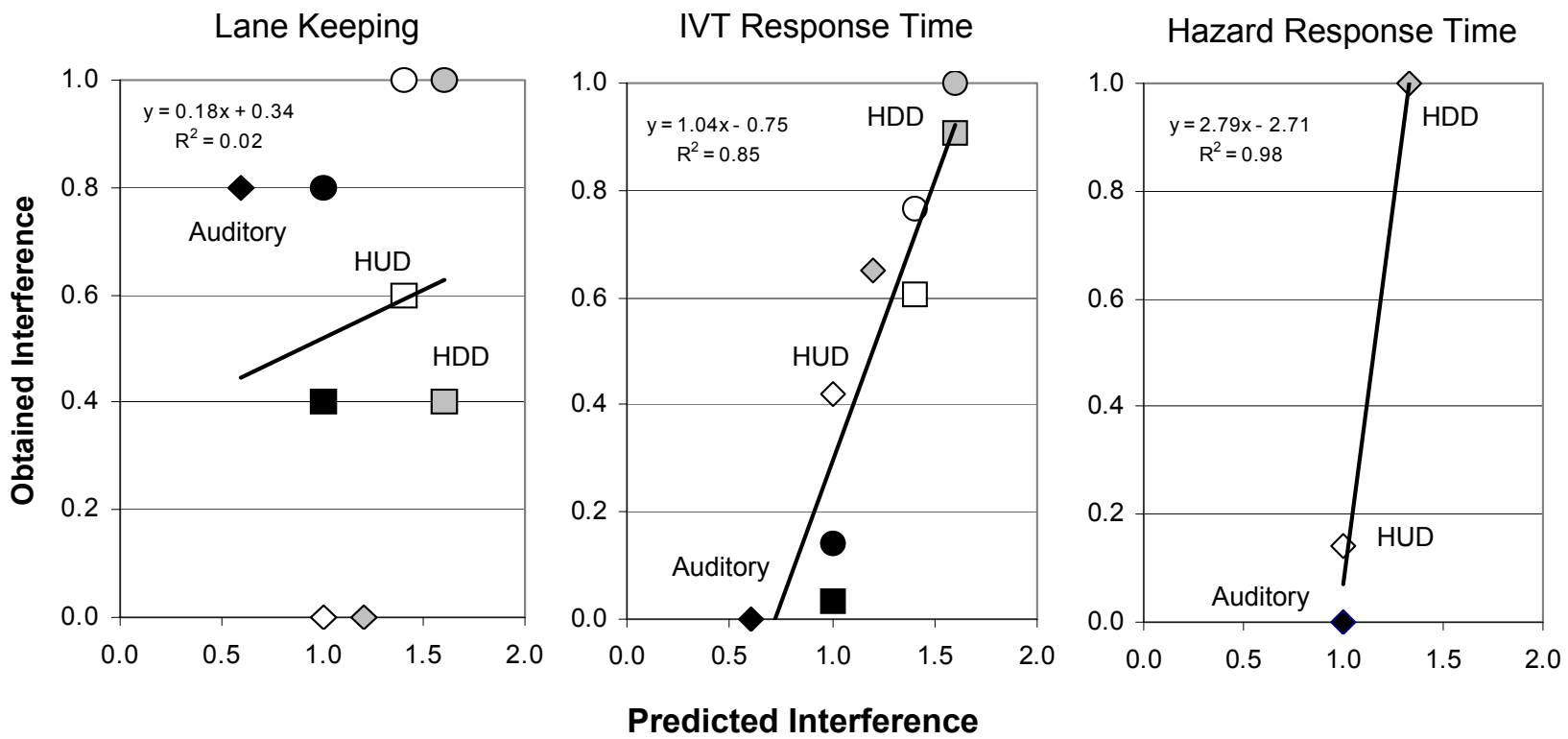

Predicted Interference

Figure 1. Obtained interference as a function of model-predicted interference for the (a) lane keeping (driving) task, (b) response times to the IVT task, and (c) hazard RTs. The HUD condition is represented by white markers, the HDD condition by light gray, and the auditory by black markers. Diamonds represent the straight rural roads, circles represent curved rural roads, and squares indicate straight urban roads.

The current model is advantageous because it is relatively simple in computation, is relatively robust (in terms of demand coding for various tasks), is theory-based, is flexible in its application, and (as shown) can make adequate performance predictions. Unfortunately, it does suffer from a few shortcomings as well; some expertise is required to establish conflict values and demand vectors. Also, the model does not output a direct measure of predicted performance losses in different dual-task conditions; rather it provides only a relative assessment of task interference between various task combinations. However, as shown in the current application, these relative interference predictions may be useful for assessing the impact of various IVTs in future automobiles and, in turn, may help validate the theoretical notions of multiple resources. 


\section{ACKNOWLEDGEMENTS}

The validation research was sponsored by a grant from General Motors (GM TCS16231 WIC). John Lenneman was the scientific / technical monitor. Special thanks to Nicholas Cassavaugh, Braden Kowitz, Hank Kaczmarski, Amy L. Alexander, and James R. Brockmole.

\section{REFERENCES}

Horrey, W.J. \& Wickens, C.D. (2002). Driving and side task performance: The effects of display clutter, separation, and modality (Technical Report No. AHFD-02-13/GM-02-2). Savoy, IL: University of Illinois, Aviation Human Factors Division.

Navon, D. \& Gopher, D. (1979). On the economy of the human processing system. Psychological Review, 86(3), 214-255.

Wickens, C.D. (2002). Multiple resources and performance prediction. Theoretical Issues in Ergonomics Science, 3(2), 159-177. 